\title{
COVID-19 Vaccination for Endocrine Patients: A Position Statement from the Korean Endocrine Society
}

Cheol Ryong $\mathrm{Ku}^{1}$, Kyong Yeun Jung ${ }^{2}$, Chang Ho Ahn ${ }^{3}$, Jun Sung Moon ${ }^{4}$, Ju Hee Lee ${ }^{5}$, Eun Heui Kim ${ }^{6}$, Hyemi Kwon ${ }^{7}$, Hee Kyung Kim ${ }^{8}$, Sunghwan Suh $^{9}$, Sangmo Hong ${ }^{10}$, Jeonghoon $\mathrm{Ha}^{11}$, Eun Roh ${ }^{12}$, Jin Hwa Kim ${ }^{13}$, Mi-kyung $\mathrm{Kim}^{14}$, the Committee of Clinical Practice Guideline of the Korean Endocrine Society

${ }^{1}$ Department of Internal Medicine, Severance Hospital, Yonsei University College of Medicine; ${ }^{2}$ Department of Internal Medicine, Nowon Eulji Medical Center, Eulji University, Seoul; ${ }^{3}$ Department of Internal Medicine, Seoul National University Bundang Hospital, Seoul National University College of Medicine, Seongnam; ${ }^{4}$ Department of Internal Medicine, Yeungnam University Medical Center, Yeungnam University College of Medicine, Daegu; ${ }^{5}$ Department of Internal Medicine, Chungnam National University Hospital, Chungnam National University College of Medicine, Daejeon; ${ }^{6}$ Department of Internal Medicine, Pusan National University Hospital, Pusan National University School of Medicine and Biomedical Research Institute, Busan; ${ }^{7}$ Department of Internal Medicine, Kangbuk Samsung Hospital, Sungkyunkwan University School of Medicine, Seoul; ${ }^{8}$ Department of Internal Medicine, Chonnam National University Hwasun Hospital, Chonnam National University Medical School, Hwasun; ${ }^{9}$ Department of Internal Medicine, Dong-A University Medical Center, Dong-A University College of Medicine, Busan; ${ }^{10}$ Department of Internal Medicine, Hanyang University Guri Hospital, Hanyang University College of Medicine, Guri; 'Division of Endocrinology and Metabolism, Department of Internal Medicine, Seoul St. Mary's Hospital, College of Medicine, The Catholic University of Korea; ${ }^{12}$ Division of Endocrinology and Metabolism, Department of Internal Medicine, Korea University College of Medicine, Seoul; ${ }^{13}$ Department of Internal Medicine, Chosun University Hospital, Chosun University College of Medicine, Gwangju; ${ }^{14}$ Department of Internal Medicine, Inje University Haeundae Paik Hospital, Inje University College of Medicine, Busan, Korea

Since the first outbreak of coronavirus disease 2019 (COVID-19), ongoing efforts have been made to discover an efficacious vaccine against COVID-19 to combat the pandemic. In most countries, both mRNA and DNA vaccines have been administered, and their side effects have also been reported. The clinical course of COVID-19 and the effects of vaccination against COVID-19 are both influenced by patients' health status and involve a systemic physiological response. In view of the systemic function of endocrine hormones, endocrine disorders themselves and the therapeutics used to treat them can influence the outcomes of vaccination for COVID-19. However, there are very limited data to support the development of clinical guidelines for patients with specific medical backgrounds based on large clinical trials. In the current severe circumstances of the COVID-19 pandemic, position statements made by clinical specialists are essential to provide appropriate recommendations based on both medical evidence and clinical experiences. As endocrinologists, we would like to present the medical background of COVID-19 vaccination, as well as precautions to prevent the side effects of COVID-19 vaccination in patients with specific endocrine disorders, including adrenal insufficiency, diabetes mellitus, osteoporosis, autoimmune thyroid disease, hypogonadism, and pituitary disorders.

Keywords: Adrenal insufficiency; Thyroiditis, autoimmune; COVID-19 vaccines; Diabetes mellitus; Osteoporosis; Hypogonadism; Hypopituitarism; Pituitary neoplasms

Received: 19 July 2021, Revised: 2 August 2021, Accepted: 5 August 2021

Corresponding author: Mi-kyung Kim

Department of Internal Medicine, Inje University Haeundae Paik Hospital, Inje University College of Medicine, 875 Haeun-daero, Haeundae-gu, Busan 48108, Korea

Tel: +82-51-797-2060, Fax: +82-51-797-2070, E-mail: kmkdoc@paik.ac.kr

\section{Copyright $\odot 2021$ Korean Endocrine Society}

This is an Open Access article distributed under the terms of the Creative Commons Attribution Non-Commercial License (https://creativecommons.org/ licenses/by-nc/4.0/) which permits unrestricted non-commercial use, distribution, and reproduction in any medium, provided the original work is properly cited. 


\section{INTRODUCTION}

Coronavirus disease 2019 (COVID-19) is a multi-factorial disease that affects nearly all the organ systems in the patient's body. This viral infection promotes chronic systemic inflammation due to the release of cytokines and abnormal activation of the innate immune system. In the era of the COVID-19 pandemic, the rapid and widespread implementation of vaccination is critical to limit the current outbreak [1].

The discovery of an efficacious vaccine for COVID-19 will be a giant step in the fight against this pandemic. The primary analysis results of the first two vaccines approved by the U.S. Food and Drug Administration (FDA), Pfizer/BioNTech, and Moderna, were reported in late 2020 [2,3]. Patients with malignancies, chronic lung disease, cardiac disease, and immunosuppressive conditions, as well as endocrine disorders such as diabetes and obesity, were included in these trials. Efficacy and safety were comparable in patients with endocrine disorders and healthy subjects. There have been no reports of any specific side effects or reduced efficacy of COVID-19 vaccination in patients with endocrine disorders. Thus, several clinical societies have suggested that patients with stable endocrine disorders should follow the same recommendations as the general population [4-6].

From a clinical standpoint, the safety issues of COVID-19 vaccination are important for both clinicians and patients with endocrine disorders. Hormonal abnormalities and the therapeutics used to treat them can influence the outcomes and side effects of COVID-19 vaccination. In this position statement, we present the precautions and safety issues of COVID-19 vaccination in patients with endocrine disorders, including adrenal disorders, diabetes mellitus, osteoporosis, thyroid disease, hypogonadism, and pituitary disorders.

\section{ADRENAL DISEASE}

The adrenal gland produces various steroid hormones and catecholamines. Steroid hormones, particularly glucocorticoids, play a pivotal role in the modulation of the immune system. Thus, adrenal diseases can be associated with immune dysfunction, which may increase susceptibility to COVID-19. Due to the immunosuppressive effects of cortisol, patients with active Cushing's syndrome are susceptible to various infections. This point also applies to COVID-19 infection. In a report from Italy, patients with active Cushing's syndrome showed a more severe clinical presentation and a higher rate of COVID-19 infection than the general population [7]. Patients who are taking a high dose of a glucocorticoid as an immunosuppressive treatment are also at an increased risk of severe COVID-19 infection [8,9].

Patients with adrenal insufficiency have an increased risk of infection due to impaired innate immunity. In addition, patients with adrenal insufficiency have an increased risk of adrenal crisis, which is a serious complication that may lead to mortality $[10,11]$. Considering the impact of adrenal diseases on the immune system and the possibility of an increased risk of COVID-19 in patients with adrenal diseases, patients with adrenal diseases should be encouraged to receive vaccinations against COVID-19. However, there is no clear evidence that patients with adrenal insufficiency are at an increased risk of COVID-19 infection. Patients with adrenal insufficiency who were adequately trained to modify their replacement therapy as appropriate had a similar rate and severity of COVID-19 infection to matched controls with intact adrenal function [12].

In addition to the general statements above, we would like to point out several special considerations about COVID-19 vaccination for patients with adrenal diseases. First, patients with adrenal insufficiency should follow "sick day rules" if they experience fever, myalgia, and chills after vaccination, which are common side effects of COVID-19 vaccination. A pre-emptive increase of the glucocorticoid dose is generally not recommended [5]. A two- or three-fold increase of the daily replacement glucocorticoid dose would suffice to manage the febrile response to COVID-19 vaccination and would probably not affect the immune response to the vaccination. If fever and myalgia persist for more than several days or clinical deterioration occurs, patients should seek medical advice from their physicians immediately. Second, it is not recommended to discontinue glucocorticoid treatment before COVID-19 vaccination for patients with adrenal insufficiency. In patients who were vaccinated against influenza, prednisolone up to $20 \mathrm{mg}$ /day did not suppress the immune response to the vaccination [13]. A physiologic glucocorticoid dose is not associated with reduced vaccination efficacy, and abrupt cessation of glucocorticoid replacement may lead to overt adrenal insufficiency. Third, currently, there is no evidence that patients with Cushing's syndrome have increased risk of vaccine-induced immune thrombotic thrombocytopenia (VITT). Although, Cushing's syndrome is a prothrombotic condition, there is no clear evidence that usual risk factors of thrombosis is associated with increased risk of VITT.

\section{DIABETES MELLITUS}

Diabetes is one of the main comorbidities closely related to the 
risk of mortality and severe clinical outcomes in patients with COVID-19 [14-18]. Numerous epidemiological findings have consistently suggested that the proportion of diabetes is higher in COVID-19 patients with a severe clinical course, and that people with diabetes are also more vulnerable to COVID-19 infection than those without diabetes [15,19-21]. Individuals with chronic disorders such as diabetes and obesity were included as study subjects in clinical trials for the approval of COVID-19 vaccines [22]. Even though detailed evidence for this category of patients is limited, it is intuitively reasonable the benefits might outweigh the risk of rare side effects that occur after vaccination.

The World Health Organization (WHO) Strategic Advisory Group of Experts (SAGE) on Immunization has recommended the use of the AZD1222 vaccine (Oxford-AstraZeneca, Cambridge, UK) in people with comorbidities including obesity and diabetes, which have been identified as increasing the risk of severe COVID-19 [23]. The Centers for Disease Control and Prevention (CDC) has stated that diabetes and obesity at any age are medical conditions that increase the risk of severe COVID-19. Considering the demand-supply matches of COVID-19 vaccines, the Advisory Committee on Immunization Practices (ACIP) advised the CDC on phased vaccine allocation (phases 1a, 1b, 1c, and 2), and phase 1c included people aged 16 to 64 years with medical conditions that increase the risk of serious complications from COVID-19 [24].

Thus, timely and appropriate vaccination is the most effective strategy for mitigating the risk of COVID-19 infection in people with diabetes. Considering the risk-benefit balance, most governmental authorities in countries including Korea [25], the United States [24], the United Kingdom [26], and European countries [27] recommend prioritizing vaccination for people with chronic disorders, including diabetes.

Impaired immunomodulation and immunodeficiency have been suggested as an explanation for why people with diabetes are vulnerable to infectious diseases, including COVID-19. Inevitably, many questions and concerns have been raised about the effects of vaccination. Clinical evidence is limited with regard to the immune response after COVID-19 vaccination, but we could refer to the evidence for other existing vaccines, such as hepatitis B, pneumococcal, and influenza postvaccine responses [28-31]. These vaccinations currently have been recommended as standard care for people with diabetes due to the considerable B-cell response [28,32]. A recent study demonstrated that the antibody response against multiple severe acute respiratory syndrome coronavirus 2 (SARS-CoV-2) antigens in patients with diabetes is superimposable, in terms of timing and titers, to that of people without diabetes and is not influenced by glucose levels [33]. These findings might justify optimistic expectations regarding the similar efficacy of COVID-19 vaccines in people with diabetes regardless of glycemic control.

The adverse effects of the current available COVID-19 vaccines are reported to be injection site pain and swelling, fatigue, fever, myalgia, and headache [22]. Little is known regarding whether vaccine-related adverse events are more prevalent in people with diabetes, although previous studies showed similar rates compared to those without diabetes [34]. These side effects are known to be mild and manageable with conservative treatment such as hydration, bed rest, and antipyretics, even in people with diabetes. Local or systemic immune reactions would lead to increased glucose levels, so more frequent blood glucose monitoring than usual might be required. In cases of fever, frequent hydration would prevent dehydration and increased blood glucose levels in people with diabetes; this strategy may be especially helpful for patients with type 1 diabetes and those receiving intensive insulin therapy. Conservative treatment according to the sick-day management guideline would be applicable to cases of postvaccine adverse events.

Although there remain efficacy and safety issues regarding COVID-19 vaccines in people with diabetes, their priority is established based on clear benefits for both individuals and society. Healthcare providers should be aware of the priority and benefits of COVID-19 vaccines in patients with diabetes and recommend prompt vaccination to them.

\section{OSTEOPOROSIS}

Based on published data, there is no evidence that bone health is directly affected by COVID-19 infection. However, the COVID-19 pandemic has reduced levels of outdoor movement, especially in older adults. On one hand, this change might lead to fewer fragility fractures in patients with osteoporosis due to a sustained reduction in the number of outdoor falls. On the other hand, a prolonged period of less outdoor movement could reduce muscle mass and decrease vitamin D levels [35], consequently worsening osteoporosis and increasing fracture risk under the circumstances of the prolonged COVID-19 pandemic. In light of these concerns, COVID-19 vaccination could positively affect patients with osteoporosis by providing them the opportunity to engage more frequently in outdoor movement. The typical side effects of COVID-19 vaccination, such as fever, chills, headache, and muscle aches, are not affected by os- 
teoporosis per se. The thrombosis with thrombocytopenia syndrome related to COVID-19 vaccination also did not seem to be directly related to osteoporosis.

There is also no evidence that pharmacological treatment for osteoporosis affects the efficacy or side-effect profile of COVID-19 vaccination. However, specific adjustments of the vaccination timing may be needed depending on the category of antiosteoporosis drug because of the similarity of side effects between anti-osteoporosis drugs and COVID-19 vaccines [36]. A 1-week interval between intravenous (IV) bisphosphonate infusion and COVID-19 vaccination is recommended to determine which intervention is responsible for acute-phase reactions such as mild fever and myalgia. It is known that flu-like symptoms appear in response to the activation of $\gamma \delta \mathrm{T}$ cells after IV bisphosphonate infusion, especially in drug-naïve patients. Both recombinant adenovirus vector-based and mRNA-based [2] COVID-19 vaccines also have acute-phase reactions such as mild fever, headache, and myalgia. A 4- to 7-day interval is recommended between the administration of other injectable antiosteoporotic agents (denosumab and romosozumab) and COVID-19 vaccination due to potential injection site reactions, such as localized pain, swelling, and/or erythema. If the COVID-19 vaccine needs to be administered simultaneously, anti-osteoporotic agents can be administered in the contralateral arm or an alternative site (abdomen or upper thigh). Meanwhile, oral bisphosphonates, raloxifene, and teriparatide should be continued without interruption or delay in patients receiving COVID-19 vaccination.

However, many clinicians reported difficulties in arranging for appropriate osteoporosis medications during the COVID-19 pandemic [37]. Delayed osteoporosis treatment can significantly increase the risk of fractures. In particular, discontinuation of denosumab, a potent antiresorptive agent, showed rapid bone loss and associated with an increased risk of fracture [38,39]. Therefore, denosumab administration should not be delayed for more than 7 months after the previous administration.

\section{THYROID DISEASE}

COVID-19 infection is known to be associated with thyroid dysfunction [6]. The possible mechanisms are the direct destruction of the thyroid gland by the SARS-CoV-2 virus, sick euthyroid syndrome, and precipitation of immune-mediated mechanisms [6]. However, thyroid disorders have been reported in rare cases after receiving the COVID-19 vaccination. Subacute thyroiditis and Graves' disease were reported following
COVID-19 vaccination [11,40-42]. Although subacute thyroiditis is well known to be associated with upper respiratory viruses, rare cases were reported with traditional inactivated viral vaccines or live-attenuated vaccines such as influenza vaccines $[11,40]$. The five patients with subacute thyroiditis following the COVID-19 vaccine were managed with glucocorticoids, non-steroidal anti-inflammatory drugs, or beta-blockers [11, $40,41]$. Vaccines have been found to trigger an immune response that may cause autoimmune disorders, including autoimmune thyroid disease [42]. A possible mechanism is that the viral protein concentration reaches a peak in 24 to 48 hours and an autoimmune response could be triggered [43]. A study reported that two healthy women who received COVID-19 vaccines were diagnosed with Graves' disease [42]. Their thyroid hormone levels increased, thyroid-stimulating hormone levels were suppressed, and anti-thyroid antibody levels increased within a few days [42]. The patients were treated with anti-thyroid drugs and had an adequate response [42].

However, COVID-19 vaccination is not contraindicated in patients with autoimmune thyroid diseases such as Hashimoto's thyroiditis and Graves' disease. The American Thyroid Association and European Thyroid Association noticed that patients with thyroid disorders, such as autoimmune thyroid disease and thyroid cancer, should receive the COVID-19 vaccination if they are medically stable $[4,12]$. If a patient is medically unstable, it should be judged on a case-by-case basis by the physician. The present vaccines have been evaluated and approved for emergency use in the United States by the FDA. Clinical trials of the COVID-19 vaccines have enrolled thousands of participants, some of whom had thyroid disease. Moreover, more than 1 billion people have been vaccinated for COVID-19 with various vaccines. However, thyroid disease has not been found to be associated with an increased risk of vaccination-related complications. COVID-19 vaccination is advantageous for patients with thyroid disease, and although it might cause thyroid disease in some cases, those cases are also treatment-responsive.

\section{HYPOGONADISM}

The available evidence suggests that males are more susceptible to severe COVID-19 than females, resulting in a higher mortality rate [44]. Data have also shown that significantly more men were hospitalized for COVID-19 than their female counterparts [45]. Therefore, male sex itself could be considered a risk factor for severe COVID-19. 
Given the disparities of clinical outcomes between men and women, the impact of biological sex on the immune response against COVID-19 infection has become an area of interest. Sex-specific differences in the immune responses of males and females have an impact on the outcome of infectious diseases such as COVID-19 [46]. The sex hormones (estrogens and androgens) have been mentioned as underlying factors responsible for the differential immune response to COVID-19 [45]. Estrogen has been reported to protect women against diseases including COVID-19 [47]. Moreover, women tend to produce more of these defensive immune-related proteins and therefore are better protected against infection, which leads to better immune protection against COVID-19 [45]. Physiological mechanisms that are likely to play a role in sex-specific differences in COVID-19 outcome include the entry of the virus into the body and immune differences [48]. In addition, immune responses and the cytokine storm, which is one of the pathophysiological features of severe COVID-19, appear to differ between men and women [46]. In contrast, androgens have been implicated in the overactivation of immune cells, cytokine storm, and the attendant severe inflammation, which may predispose males to severe COVID-19 [45]. It has been suggested that estrogen protects females against severe COVID-19, while androgens worsen COVID-19 outcomes in males [49]. It is also important to consider potential confounders such as behavioral differences and societal factors (e.g., higher rates of smoking in men) when evaluating COVID-19 case-fatality rate disparities according to sex $[6,50]$.

The effect of androgens on COVID-19 outcomes was investigated in Italy [51]. In that study, patients with prostate cancer undergoing treatment with androgen deprivation therapy (ADT) showed a significantly lower risk of severe COVID-19 than the non-ADT group. Based on those results, it can be postulated that a high androgen concentration exacerbates the severity of COVID-19 and that ADT could be suggested to protect men from severe COVID-19. However, future research is needed to examine whether hormone replacement therapy may have a therapeutic role in treating patients diagnosed with COVID-19.

Because of their stronger immune response, as discussed above, women are known to report more side effects after COVID-19 vaccination [52]. However, this heightened response in women seems to be correlated with the development of higher levels of antibodies against COVID-19. Considering the high morbidity and mortality from COVID-19 infection, the benefits of vaccination in patients with hypogonadism outweigh the risk of anaphylaxis or other side effects, which are treatable.
In view of the robust data regarding the role of sex hormones in immune response, specifically the anti-inflammatory effects and the anabolic effect [6], it is important to consider their potential role of finding high-risk patients or developing treatment strategies for patients with COVID-19, particularly older adults and those with hormone deficiencies. COVID-19 vaccination is advantageous for these high-risk patients. Future research is needed to examine whether hormone replacement therapy may have a therapeutic role in treating patients diagnosed with COVID-19.

\section{PITUITARY DISEASE}

The COVID-19 vaccination data did not report any side effects or specific contraindications related to pituitary disease [3]. Therefore, COVID-19 vaccination is recommended in patients with stable pituitary disease as much as in the general population. However, the safety of COVID-19 vaccination in patients with progressive or untreated pituitary disease has not been investigated.

The side effects of COVID-19 vaccines include pain at the injection site, fatigue, headache, myalgia, joint pain, chills, and fever [2,3]. Due to these side effects, additional glucocorticoid doses may be necessary for patients with adrenal insufficiency. The Pituitary Society conducted a survey on glucocorticoid therapy in adrenal insufficiency patients who received COVID-19 vaccines, and they obtained 103 responses from 273 members [5]. Most clinicians have indicated their plans to maintain the current glucocorticoid dose for patients receiving the COVID-19 vaccination. However, if fever, joint, or muscle pain occurred, they would respond by increasing the glucocorticoid dose [5]. Concerns about COVID-19 outweigh the adverse effects of vaccination in patients with adrenal insufficiency. Several studies have reported that patients with adrenal insufficiency had a higher risk of infection than the general population [1]. Patients with adrenal insufficiency have an impaired innate immune response. Since the immune defense against infection is blunted, they are susceptible to infection [53]. Therefore, the risk of death from COVID-19 infection was reported to be high in patients with adrenal insufficiency [1]. Although there is still no definitive evidence, expert opinions have recommended that patients with adrenal insufficiency should be fully informed regarding the side-effects of COVID-19 vaccination and the sick day rules in case they develop a fever. 


\section{CONCISE SUMMARY}

1. In patients with stable adrenal disease, COVID-19 vaccines should be administered according to the same standards as the general population.

2. In patients with adrenal insufficiency, COVID-19 vaccination is recommended similarly to the healthy general population. In addition, there is no need to discontinue or change the dose of steroid medication due to vaccination. However, if fever, severe muscle pain, and chills occur after vaccination, the steroid dose should be increased by two to three times according to the sick day rules.

3. In patients with diabetes, COVID-19 vaccination should be administered as a priority regardless of the diabetes type.

4. In patients with diabetes, blood glucose monitoring is required more often than usual for several days after vaccination. If a patient's blood glucose is not controlled adequately, appropriate management should be provided.

5. In patients with osteoporosis, COVID-19 vaccination should be administered according to the same standards as the general population.

6. To differentiate the side effects of COVID-19 vaccination and intravenous bisphosphonate, a 1-week interval is recommended.

7. Due to the potential injection site reaction, a 4- to 7-day interval for injectable anti-osteoporotic agents (denosumab and romosozumab) is recommended. Meanwhile, oral bisphosphonates, raloxifene and teriparatide should be continued without interruption or delay in patients receiving COVID-19 vaccination.

8. In patients with thyroid dysfunction, COVID-19 vaccination should be administered according to the same standards as the general population.

9. In patients with hypogonadism, COVID-19 vaccination should be administered according to the same standards as the general population.

10. In patients with stable pituitary disease, COVID-19 vaccination should be administered according to the same standards as the general population.

11. In patients taking glucocorticoids due to secondary adrenal insufficiency, the recommendation for COVID-19 vaccination is the same as concise summary no. 2 .

\section{CONCLUSIONS}

Most patients with endocrinopathy must receive COVID-19 vaccination because COVID-19 infection induces an increased prevalence or severity of the complications caused by endocrine disorders. Furthermore, there is no clinical evidence that hormonal replacement reduces the efficacy or increases the side effects of COVID-19 vaccines. There is no clinical evidence that the type of COVID-19 vaccine is associated with differences in the development of endocrinopathy-associated complications.

\section{CONFLICTS OF INTEREST}

No potential conflict of interest relevant to this article was reported.

\section{ORCID}

Cheol Ryong Ku https://orcid.org/0000-0001-8693-9630

Mi-kyung Kim https://orcid.org/0000-0003-1111-9122

\section{REFERENCES}

1. Rhee EJ, Kim JH, Moon SJ, Lee WY. Encountering COVID-19 as endocrinologists. Endocrinol Metab (Seoul) 2020; 35:197-205.

2. Baden LR, El Sahly HM, Essink B, Kotloff K, Frey S, Novak R, et al. Efficacy and safety of the mRNA-1273 SARSCoV-2 vaccine. N Engl J Med 2021;384:403-16.

3. Polack FP, Thomas SJ, Kitchin N, Absalon J, Gurtman A, Lockhart S, et al. Safety and efficacy of the BNT162b2 mRNA COVID-19 vaccine. N Engl J Med 2020;383:260315.

4. Luger A, Giustina A, Peeters R. European Society of Endocrinology (ESE)'s statement concerning COVID 19 vaccination: 'follow the same recommendations for patients with stable endocrine disorders as for the general population' 2021 [Internet]. Bristol: European Society of Endocrinology; 2021 [cited 2021 Aug 9]. Available from: https://www.ese-hormones.org/ news/ese-news/european-society-of-endocrinology-eses-statement-concerning-covid-19-vaccination-follow-thesame-recommendations-for-patients-with-stable-endocrinedisorders-as-for-the-general-population.

5. Katznelson L, Gadelha M. Glucocorticoid use in patients with adrenal insufficiency following administration of the COVID-19 vaccine: a pituitary society statement. Pituitary 2021;24:143-5.

6. Puig-Domingo M, Marazuela M, Yildiz BO, Giustina A. COVID-19 and endocrine and metabolic diseases. An up- 
dated statement from the European Society of Endocrinology. Endocrine 2021;72:301-16.

7. Serban AL, Ferrante E, Carosi G, Indirli R, Arosio M, Mantovani G. COVID-19 in Cushing disease: experience of a single tertiary centre in Lombardy. J Endocrinol Invest 2021; 44:1335-6.

8. Adir Y, Humbert M, Saliba W. COVID-19 risk and outcomes in adult asthmatic patients treated with biologics or systemic corticosteroids: nationwide real-world evidence. J Allergy Clin Immunol 2021;148:361-7.

9. Brenner EJ, Ungaro RC, Gearry RB, Kaplan GG, KissousHunt M, Lewis JD, et al. Corticosteroids, but not TNF antagonists, are associated with adverse COVID-19 outcomes in patients with inflammatory bowel diseases: results from an international registry. Gastroenterology 2020;159:481-91.

10. Arlt W, Baldeweg SE, Pearce SHS, Simpson HL. Endocrinology in the time of COVID-19: management of adrenal insufficiency. Eur J Endocrinol 2020;183:G25-32.

11. Stewart PM, Biller BM, Marelli C, Gunnarsson C, Ryan MP, Johannsson G. Exploring inpatient hospitalizations and morbidity in patients with adrenal insufficiency. J Clin Endocrinol Metab 2016;101:4843-50.

12. Carosi G, Morelli V, Del Sindaco G, Serban AL, Cremaschi A, Frigerio S, et al. Adrenal insufficiency at the time of COVID-19: a retrospective study in patients referring to a tertiary center. J Clin Endocrinol Metab 2021;106:e1354-61.

13. Inoue S, Shibata Y, Takabatake N, Igarashi A, Abe S, Kubota I. Influence of corticosteroid therapy on the serum antibody response to influenza vaccine in elderly patients with chronic pulmonary diseases. EXCLI J 2013;12:760-5.

14. Chung SM, Lee YY, Ha E, Yoon JS, Won KC, Lee HW, et al. The risk of diabetes on clinical outcomes in patients with coronavirus disease 2019: a retrospective cohort study. Diabetes Metab J 2020;44:405-13.

15. Gregory JM, Slaughter JC, Duffus SH, Smith TJ, LeStourgeon LM, Jaser SS, et al. COVID-19 severity is tripled in the diabetes community: a prospective analysis of the pandemic's impact in type 1 and type 2 diabetes. Diabetes Care 2021;44:526-32.

16. Huang I, Lim MA, Pranata R. Diabetes mellitus is associated with increased mortality and severity of disease in COVID-19 pneumonia: a systematic review, meta-analysis, and meta-regression. Diabetes Metab Syndr 2020;14:395-403.

17. Kim MK, Jeon JH, Kim SW, Moon JS, Cho NH, Han E, et al. The clinical characteristics and outcomes of patients with moderate-to-severe coronavirus disease 2019 infection and diabetes in Daegu, South Korea. Diabetes Metab J 2020;44: 602-13.

18. Lim S, Bae JH, Kwon HS, Nauck MA. COVID-19 and diabetes mellitus: from pathophysiology to clinical management. Nat Rev Endocrinol 2021;17:11-30.

19. Barron E, Bakhai C, Kar P, Weaver A, Bradley D, Ismail H, et al. Associations of type 1 and type 2 diabetes with COVID-19-related mortality in England: a whole-population study. Lancet Diabetes Endocrinol 2020;8:813-22.

20. Cariou B, Hadjadj S, Wargny M, Pichelin M, Al-Salameh A, Allix I, et al. Phenotypic characteristics and prognosis of inpatients with COVID-19 and diabetes: the CORONADO study. Diabetologia 2020;63:1500-15.

21. Moon SJ, Rhee EJ, Jung JH, Han KD, Kim SR, Lee WY, et al. Independent impact of diabetes on the severity of coronavirus disease 2019 in 5,307 patients in South Korea: a nationwide cohort study. Diabetes Metab J 2020;44:737-46.

22. Pal R, Bhadada SK, Misra A. COVID-19 vaccination in patients with diabetes mellitus: current concepts, uncertainties and challenges. Diabetes Metab Syndr 2021;15:505-8.

23. World Health Organization. Interim recommendations for use of the AZD1222 (ChAdOx1-S [recombinant]) vaccine against COVID- 19 developed by Oxford University and AstraZeneca [Internet]. Geneva: WHO; 2021 [cited 2021 Aug 9]. Available from: https://apps.who.int/iris/bitstream/handle/10665/340920/ WHO-2019-nCoV-vaccines-SAGE_recommendationAZD1222-2021.2-eng.pdf? sequence $=1$ \&isAllowed $=\mathrm{y}$.

24. Dooling K, Marin M, Wallace M, McClung N, Chamberland $\mathrm{M}$, Lee GM, et al. The advisory committee on immunization practices' updated interim recommendation for allocation of COVID-19 vaccine: United States, December 2020. MMWR 2021;69:1657-60.

25. Central Disaster Management Headquarters. Who first gets the COVID-19 vaccination? [Internet]. Cheongju: Korea Disease Control and Prevention Agency; 2021 [cited 2021 Aug 9]. Available from: https://ncv.kdca.go.kr/menu. es? $\mathrm{mid}=\mathrm{a} 10117010000$.

26. Diabetes UK. Coronavirus vaccines and diabetes [Internet]. London: Diabetes UK; 2021 [cited 2021 Aug 9]. Available from: https://www.diabetes.org.uk/about_us/news/coronavirusvaccines.

27. European Centre for Disease Prevention and Control. COVID-19 vaccination and prioritisation strategies in the EU/ EEA. Stockholm: ECDC; 2020.

28. Frasca D, Diaz A, Romero M, Mendez NV, Landin AM, Ryan JG, et al. Young and elderly patients with type 2 diabe- 
tes have optimal B cell responses to the seasonal influenza vaccine. Vaccine 2013;31:3603-10.

29. Li Volti S, Caruso-Nicoletti M, Biazzo F, Sciacca A, Mandara G, Mancuso M, et al. Hyporesponsiveness to intradermal administration of hepatitis B vaccine in insulin dependent diabetes mellitus. Arch Dis Child 1998;78:54-7.

30. Looijmans-Van den Akker I, Verheij TJ, Buskens E, Nichol KL, Rutten GE, Hak E. Clinical effectiveness of first and repeat influenza vaccination in adult and elderly diabetic patients. Diabetes Care 2006;29:1771-6.

31. Smith SA, Poland GA. Use of influenza and pneumococcal vaccines in people with diabetes. Diabetes Care 2000;23:95108.

32. American Diabetes Association. 4. Comprehensive medical evaluation and assessment of comorbidities: standards of medical care in diabetes-2021. Diabetes Care 2021;44(Suppl 1):S40-52.

33. Lampasona V, Secchi M, Scavini M, Bazzigaluppi E, Brigatti C, Marzinotto I, et al. Antibody response to multiple antigens of SARS-CoV-2 in patients with diabetes: an observational cohort study. Diabetologia 2020;63:2548-58.

34. Kesavadev J, Misra A, Das AK, Saboo B, Basu D, Thomas $\mathrm{N}$, et al. Suggested use of vaccines in diabetes. Indian J Endocrinol Metab 2012;16:886-93.

35. Ulivieri FM, Banfi G, Camozzi V, Colao A, Formenti AM, Frara S, et al. Vitamin D in the COVID-19 era: a review with recommendations from a G.I.O.S.E.G. expert panel. Endocrine 2021;72:597-603.

36. Zhu FC, Li YH, Guan XH, Hou LH, Wang WJ, Li JX, et al. Safety, tolerability, and immunogenicity of a recombinant adenovirus type-5 vectored COVID-19 vaccine: a dose-escalation, open-label, non-randomised, first-in-human trial. Lancet 2020;395:1845-54.

37. Fuggle NR, Singer A, Gill C, Patel A, Medeiros A, Mlotek AS, et al. Correction to: how has COVID-19 affected the treatment of osteoporosis? An IOF-NOF-ESCEO global survey. Osteoporos Int 2021;32:801.

38. Cummings SR, Ferrari S, Eastell R, Gilchrist N, Jensen JB, McClung $\mathrm{M}$, et al. Vertebral fractures after discontinuation of denosumab: a post hoc analysis of the randomized placebo-controlled FREEDOM Trial and its extension. J Bone Miner Res 2018;33:190-8.

39. Zanchetta MB, Boailchuk J, Massari F, Silveira F, Bogado C, Zanchetta JR. Significant bone loss after stopping long-term denosumab treatment: a post FREEDOM study. Osteoporos Int 2018;29:41-7.
40. Franquemont S, Galvez J. Subacute thyroiditis after mRNA vaccine for COVID-19. J Endocr Soc 2021;5(Suppl 1):A9567.

41. Iremli BG, Sendur SN, Unluturk U. Three cases of subacute thyroiditis following SARS-CoV-2 vaccine: post-vaccination ASIA syndrome. J Clin Endocrinol Metab 2021;106: 2600-5.

42. Vera-Lastra O, Ordinola Navarro A, Cruz Domiguez MP, Medina G, Sanchez Valadez TI, Jara LJ. Two cases of Graves' disease following SARS-CoV-2 vaccination: an autoimmune/ inflammatory syndrome induced by adjuvants. Thyroid 2021 May 3 [Epub]. https://doi.org/10.1089/thy.2021.0142.

43. Dotan A, Muller S, Kanduc D, David P, Halpert G, Shoenfeld Y. The SARS-CoV-2 as an instrumental trigger of autoimmunity. Autoimmun Rev 2021;20:102792.

44. Okpechi SC, Fong JT, Gill SS, Harman JC, Nguyen TH, Chukwurah QC, et al. Global sex disparity of COVID-19: a descriptive review of sex hormones and consideration for the potential therapeutic use of hormone replacement therapy in older adults. Aging Dis 2021;12:671-83.

45. Acheampong DO, Barffour IK, Boye A, Aninagyei E, Ocansey S, Morna MT. Male predisposition to severe COVID-19: review of evidence and potential therapeutic prospects. Biomed Pharmacother 2020;131:110748.

46. Klein SL, Dhakal S, Ursin RL, Deshpande S, Sandberg K, Mauvais-Jarvis F. Biological sex impacts COVID-19 outcomes. PLoS Pathog 2020;16:e1008570.

47. Alkhouli M, Nanjundappa A, Annie F, Bates MC, Bhatt DL. Sex differences in case fatality rate of COVID-19: insights from a multinational registry. Mayo Clin Proc 2020;95:161320.

48. Qiao Y, Wang XM, Mannan R, Pitchiaya S, Zhang Y, Wotring JW, et al. Targeting transcriptional regulation of SARS-CoV-2 entry factors ACE2 and TMPRSS2. Proc Natl Acad Sci U S A 2021;118:e2021450118.

49. Ciaglia E, Vecchione C, Puca AA. COVID-19 infection and circulating ACE2 levels: protective role in women and children. Front Pediatr 2020;8:206.

50. Cai H. Sex difference and smoking predisposition in patients with COVID-19. Lancet Respir Med 2020;8:e20.

51. Montopoli M, Zumerle S, Vettor R, Rugge M, Zorzi M, Catapano $\mathrm{CV}$, et al. Androgen-deprivation therapies for prostate cancer and risk of infection by SARS-CoV-2: a populationbased study ( $\mathrm{N}=4532)$. Ann Oncol 2020;31:1040-5.

52. Shimabukuro TT, Cole M, Su JR. Reports of anaphylaxis after receipt of mRNA COVID-19 vaccines in the US-Decem- 
ber 14, 2020-January 18, 2021. JAMA 2021;325:1101-2.

53. Tresoldi AS, Sumilo D, Perrins M, Toulis KA, Prete A, Reddy $\mathrm{N}$, et al. Increased infection risk in Addison's disease and congenital adrenal hyperplasia. J Clin Endocrinol Metab 2020;105:418-29. 